\title{
New developments in the study of optical parabolic pulses in normally dispersive fibers
}

\author{
Kamal Hammani ${ }^{1}$, Brandon G. Bale ${ }^{2}$, Sonia Boscolo ${ }^{2}$, and Christophe Finot ${ }^{1}$ \\ ${ }^{1}$ Laboratoire Interdisciplinaire Carnot de Bourgogne, UMR 5209, 9 Av. A. Savary, Dijon, France \\ ${ }^{2}$ Photonics Research Group, School of Engineering and Applied Science, Aston University, Birmingham, UK \\ Christophe.Finot@u-bourgogne.fr
}

\begin{abstract}
We report two recent studies dealing with the evolution of parabolic pulses in normally dispersive fibers. On the one hand, the nonlinear reshaping from a Gaussian intensity profile towards the asymptotic parabolic shape is experimentally investigated in a Raman amplifier. On the other hand, the significant impact of the fourth order dispersion on a passive propagation is theoretically discussed: we numerically demonstrate flat-top, coherent supercontinuum generation in an all-normal dispersion-flattened photonic crystal fiber. This shape is associated to a strong reshaping of the temporal profile what becomes triangular.
\end{abstract}

Keywords: nonlinear pulse shaping, Raman amplification, self-similarity, coherent supercontinuum.

\section{INTRODUCTION}

Over the past decade there has been large interest in ultrafast optical fiber amplifiers operating in the normal dispersion regime because of the discovery that, high-energy pulses with a parabolic intensity profile and linear frequency chirp are the asymptotic solution to the system for arbitrary initial conditions [1]. These so-called "similariton" solutions propagate in a self-similar manner, holding certain relations (scaling) between pulse power, duration, and chirp parameter and can remarkably resist to the deleterious effects of optical wavebreaking, both in active and passive propagation [2]. Therefore, several applications in the field of high energy pulse generation or high-speed optical telecommunications have benefited from those properties (see for example review [3]).

In this present work, we report two new studies that further explore the dynamics of those nonlinear structures. We first describe experimental results dealing with the transition from a low-energy Gaussian pulse to the asymptotic state. The nonlinear reshaping is clearly evidenced as well as the gain saturation of the amplifier. We then discuss the impact of fourth order dispersion and we numerically demonstrate flat-top, coherent supercontinuum generation in an all-normal dispersion-flattened photonic crystal fiber. This shape is associated to a strong reshaping of the temporal profile that becomes triangular.

\section{TRANSITION DYNAMICS IN NORMALLY-DISPERSIVE OPTICAL FIBER AMPLIFIERS}

Transition dynamics of the normally-dispersive fiber amplifiers has been the subject of a limited number of theoretical works but only one experimental set of data is currently available to confirm the different mechanisms: in 2005, Billet et al. have investigated the longitudinal evolution of a picosecond pulse in an erbium-doped fiber amplifier (EDFA) [2] with special emphasis on the evolution of the wings of the pulse characteristic of the intermediate regime when plotted on a logarithmic scale [4].

Here we experimentally analyze a Raman fiber amplifier operating at normal dispersion [5] using initial lowenergy Gaussian pulses. The system under investigation is sketched in Fig. 1. The experimental set-up is based on commercially available telecommunication devices. A $10 \mathrm{GHz}$ modelocked fiber laser delivers initial picosecond hyperbolic secant pulses that are then linearly shaped to obtain 6.8 ps Gaussian pulses (such a temporal duration has been chosen in order to clearly observe the transition at the output of the fiber). The repetition rate of the source is decreased down to $5 \mathrm{GHz}$ in order to limit the depletion effects. The pulses are pre-amplified by an EDFA and an optical variable attenuator is used to vary the input power.

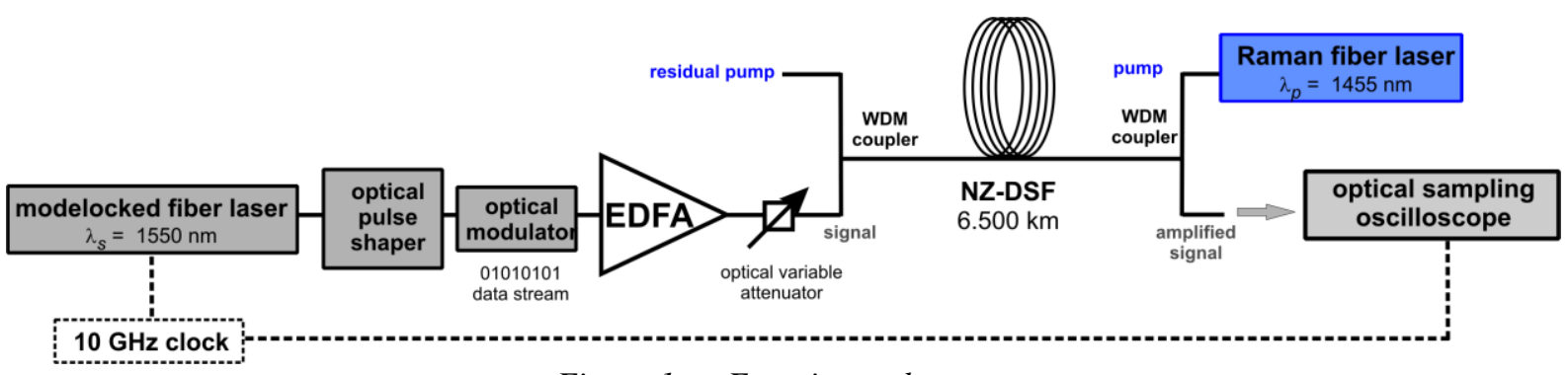

Figure 1. Experimental set-up. 
The Raman amplifier is based on a 6.1-km long non-zero dispersion shifted fiber (NZ-DSF) having a dispersion coefficient $\mathrm{D}=-2.58 \mathrm{ps} / \mathrm{km} / \mathrm{nm}$ and a nonlinear coefficient $\gamma=1.7 \mathrm{~W}^{-1} \cdot \mathrm{km}^{-1}$. The pump is a continuous wave delivered by a Raman fiber laser and a backward pumping scheme is favored to limit the deleterious transfer of the noise of pump to the signal [6]. A pumping power up to $2 \mathrm{~W}$ is used, which enables a gain in the small signal limit of $25 \mathrm{~dB}$.

Experimental temporal intensity profiles are recorded for initial peak powers ranging from 2 to $650 \mathrm{~mW}$ and are summarized in Figure 2. Results plotted on a linear scale clearly exhibit the transition from a low power Gaussian pulse affected mainly by second order dispersion towards a parabolic self-similar pulse. As the peak power grows, the nonlinearity induces a phase change that is proportional to the difference between the intensity profile and a parabolic profile. This in turn reshapes the intensity towards a parabolic form. Such an evolution has been found in agreement with an approach based on the use of a self-similar ansatz and a standard amplitude-phase decomposition [4] : reduced equations for the key pulse parameters (peak amplitude, pulse duration and chirp coefficient) along with a known hydrodynamic-type system of equations have been shown to accurately reproduce the experimental trends.
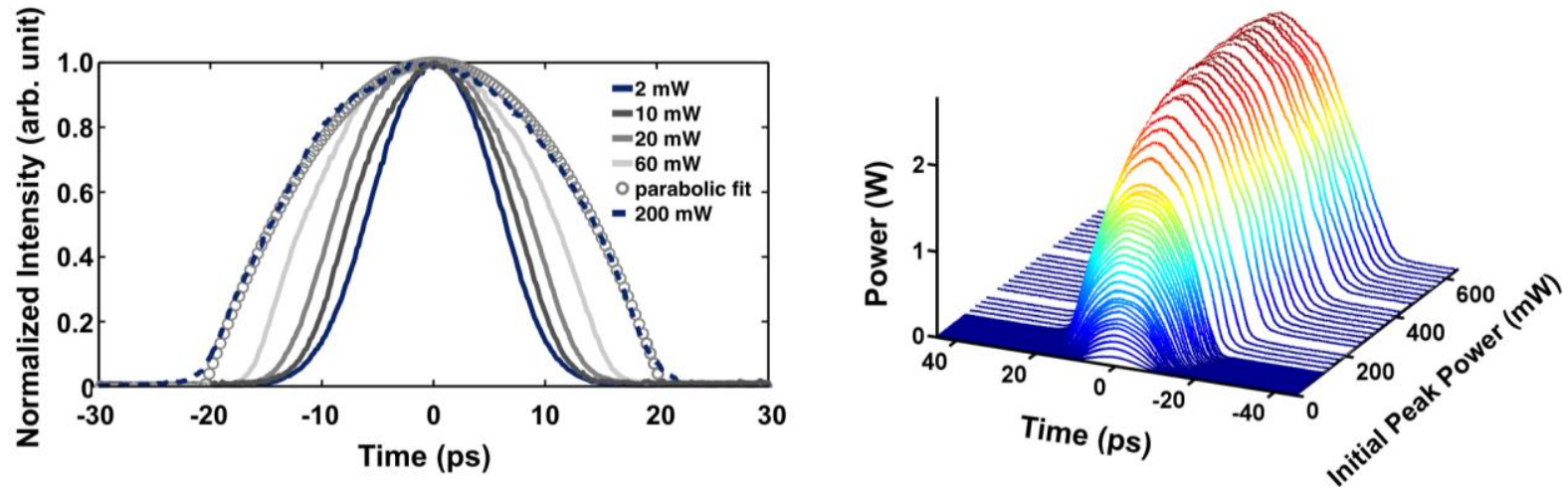

Figure 2. (a) Experimental results showing the temporal profile of output pulse for initial Gaussian pulses with varied initial peak powers. Circles represent a parabolic fit of the temporal intensity profile obtained for initial pulses having an initial peak power of $200 \mathrm{~mW}$.. (b) Results for initial peak powers up to $650 \mathrm{~mW}$.

Experimental intensity profiles have been recorded using an ultrafast optical sampling oscilloscope (ps temporal resolution).

Results also demonstrate clearly the saturation of the amplifier for initial peak-powers above $200 \mathrm{~mW}$ (the limiting factor is not here the spectral gain bandwidth but the pump energy). The output peak power saturates and does not follow anymore the typical law of the self-similar pulses. The parabolic shape is maintained with a significant increase of the temporal duration, which is in qualitative agreement with previous theoretical predictions [7, 8]. Therefore, this result constitutes the first direct experimental observation of the effect of gain saturation. Let us however note that if a co-propagating pumping scheme would have been used, longitudinal evolution would have been also affected by additional temporal depletion of the pump [5].

\section{IMPACT OF FOURTH-ORDER DISPERSION ON PASSIVE PROPAGATION}

The spectral dispersion profile of a fiber is known to be a critical parameter for the generation of broadband light sources. The broadest spectra are generated when pumping occurs in the anomalous dispersion regime of the fiber. However, this results in decreased spectral coherence and flatness, especially when picosecond pump pulses are used. Previous attempts have been made to overcome this drawback by using dispersion-flattened fibers continuously tapered from anomalous to normal dispersion. However, due to limited availability of such fiber tapers, there is a recent renewed interest to consider fibers with longitudinally constant dispersion profiles, where a sufficiently broad, stable and flat-top spectrum can still be achieved $[9,10]$.

We propose here a new method for generating coherent and flat-top SC spectra which relies on the effect of fourth-order dispersion (FOD) on parabolically-shaped pulses in an all-normal dispersion (ANDi) -flattened PCF. We numerically demonstrate that compact and remarkably flat spectra can be achieved while a triangular temporal profile is formed.

For our numerical simulations we use an ANDi PCF with flattened dispersion characteristics around $1060 \mathrm{~nm}$, similar to what has been demonstrated in [11] (Fig. 3a). The third-order dispersion, which can degrade the selfsimilar pulse propagation [12] is zero at the pump wavelength. Pulse propagation in the PCF is described by the 
standard nonlinear Schrödinger equation including the effects of second-order dispersion, FOD and Kerr nonlinearity. Linearly chirped parabolic pulses with pulse energy $12 \mathrm{~nJ}$, full-width half-maximum $2.6 \mathrm{ps}$ and chirp parameter $3.35 \mathrm{THz}^{2}$ generated in an ytterbium-doped fibre amplifier [1] were used as initial conditions for pulse propagation in the PCF.
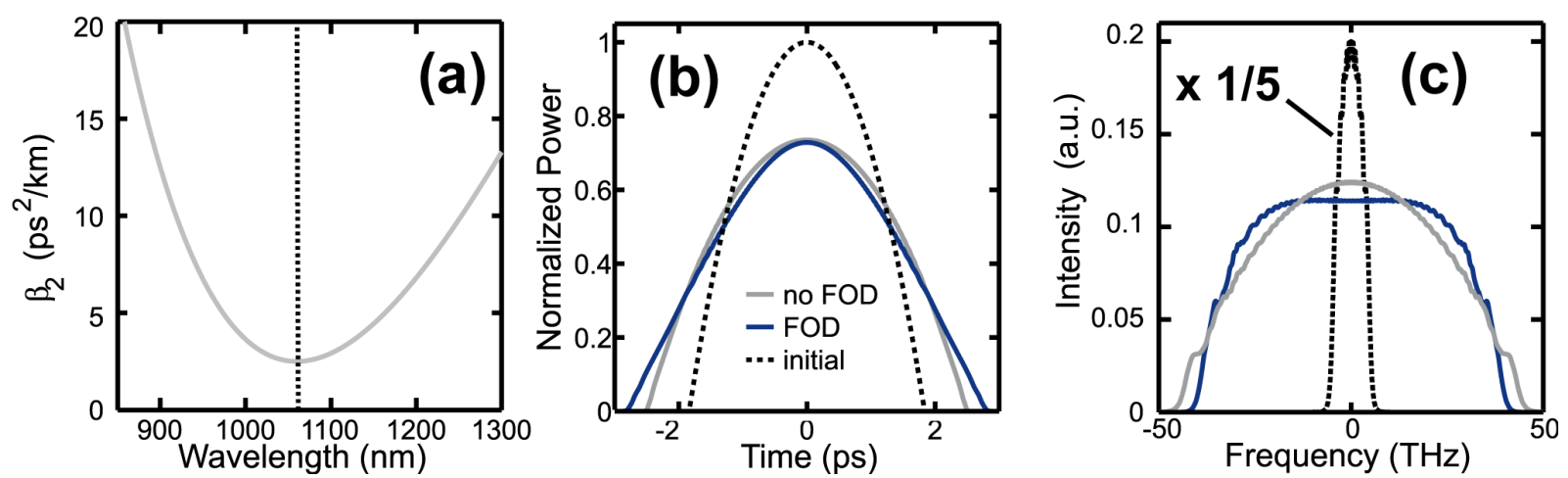

Figure 3. (a) Dispersion profile of the PCF considered. (b) Temporal and (c) spectral power after propagation distance of $1.5 \mathrm{~m}$ with (blue solid line) and without (grey solid line) FOD. Initial pulse is plotted with a dotted black line.

To examine the impact of FOD we compare the evolution obtained in the concave dispersion-profiled PCF to that occurring in an equivalent fiber with spectrally constant dispersion $\left(\beta_{4}=0\right)$. The temporal and spectral profiles after a propagation distance of $1.5 \mathrm{~m}$ are shown in Fig. 3(b-c). We can see that in the absence of FOD, the pulse evolves self-similarly maintaining its parabolic shape both in the time and frequency domains, while undergoing a continuous, nonlinearity-dominated temporal and spectral broadening (Fig. 4(a-c)) [13]. These results are consistent with the well-known, intrinsic resistance of similaritons to the optical wave breaking phenomenon $[1,3]$. In the presence of FOD, significant temporal and spectral reshaping of the parabolic pulse occurs over short propagation distances. In the temporal domain, the wings of the pulse, where the outermost instantaneous frequencies are located (given the linear chirp), experience enhanced broadening due to the large local dispersion. This effect generates a triangular shape which is maintained over a non-negligible propagation distance. The use of the FOD effect in a normally dispersive nonlinear fiber thus provides a new, alternative passive method for generating triangular-shaped pulses with respect to that introduced in [14].

In the spectral domain, the power after $1.5 \mathrm{~m}$ propagation has very rapidly falling edges and a ripple-free top over $50 \mathrm{THz}(190 \mathrm{~nm})$ span (Fig. 3(c)). However, this flat-top spectrum is only a transient state and for further propagation distance the spectral power evolves into an M-shape (Fig. 4(d)). This typical shape can be linked to the self-phase modulation induced by the triangular temporal intensity profile. In the presence of FOD, the spectral bandwidth stabilizes more rapidly, typically leading to narrower spectrums since the pulse experiences both enhanced temporal broadening peak power reduction.
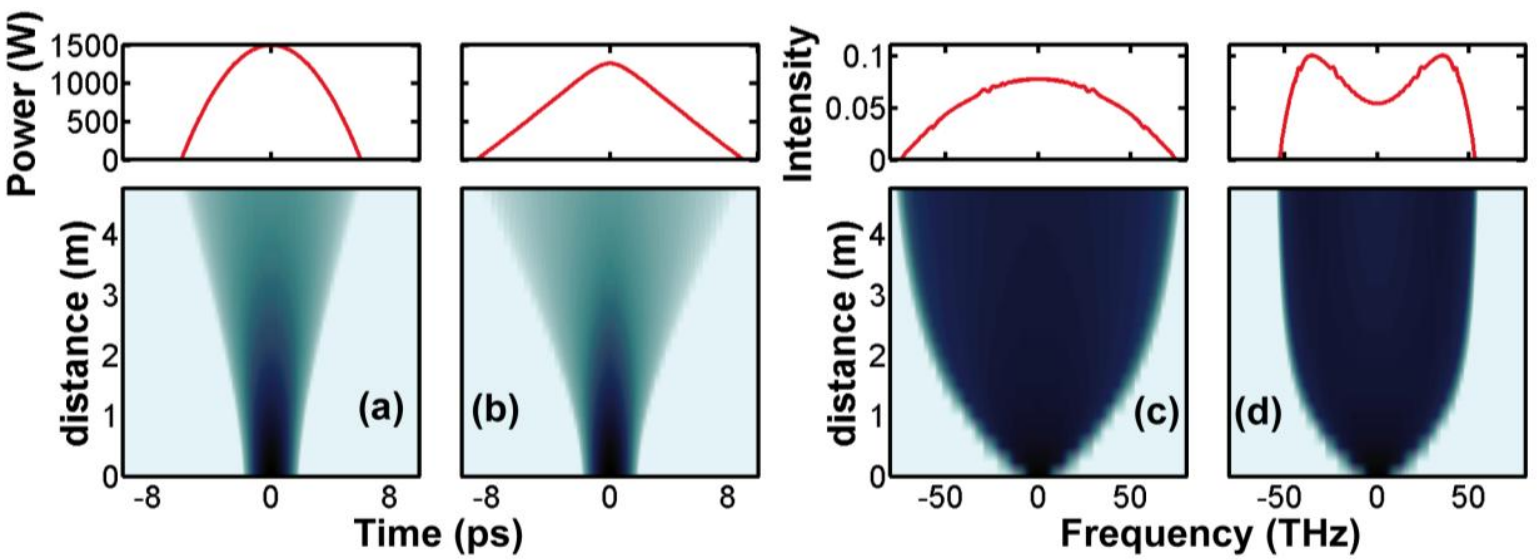

Figure 4. Longitudinal temporal (a-b) and spectral $(\boldsymbol{c}-\boldsymbol{d})$ evolutions of a parabolic pulse without $(\boldsymbol{a}, \boldsymbol{c})$ and with $(\boldsymbol{b}, \boldsymbol{d})$ FOD. 


\section{CONCLUSIONS}

In this report we have studied two issues dealing directly with the specific features of optical parabolic pulses evolving in normally dispersive fibers. On the one hand, we have focused on the transition from low-energy Gaussian pulses to parabolic pulses in a Raman amplifier. A detailed experimental study coupled with a theoretical analysis highlights the various stages of the nonlinear reshaping. Further, the impact of the energy saturation of the amplifier is also clearly shown. On the other hand, we have proposed a new method for generating coherent, ultra-flat SC spectra based on the effect of the FOD on picosecond parabolic seed pulses in an ANDi PCF. We have numerically shown that compact spectra with ripple-free top over approximately 190 $\mathrm{nm}$ wavelength range can be achieved, while triangular pulses are formed in the time domain. We have also checked that the use of initial parabolic pulses provides substantially better performance as compared to conventional Gaussian pulses. These two studies and other recent results dealing with improved spectral compression [15] demonstrate that even though optical parabolic pulses have been highlighted as soon as 2000, there are still some aspects of the nonlinear pulse shaping that are not fully explored and that many applications, such as optical pulse processing, may benefit from a better understanding.

\section{ACKNOWLEDGEMENTS}

The authors would like to acknowledge support from the Engineering and Physical Sciences Research Council (Grant No. EP/FO2956X/1), the British Council (Alliance Grant No. 10.002), the Region Bourgogne (PARI PHOTCOM), the Agence Nationale de la Recherche (ANR SOFICARS, ANR-07-RIB-013-03) and the Ministère des Affaires étrangères et européenne (Alliance grant 22836ZJ).

\section{REFERENCES}

[1] M. E. Fermann, et al., "Self-similar propagation and amplification of parabolic pulses in optical fibers," Phys. Rev. Lett., vol. 84, pp. 6010-6013, 6/2000 2000.

[2] J. M. Dudley, et al., "Self-similarity in ultrafast nonlinear optics," Nat. Phys., vol. 3, pp. 597-603, 9/2007 2007.

[3] C. Finot, et al., "Optical parabolic pulse generation and applications," IEEE J. Quantum Electron., vol. 45, pp. 1482-1489, 2009.

[4] S. Boscolo, et al., "Self-similar parabolic optical solitary waves," Theor. Math. Phys., vol. 133, pp. $1647-1656,20022002$.

[5] C. Finot, "Influence of the pumping configuration on the generation of optical similaritons in optical fibers," Opt. Commun., vol. 249, pp. 553-561, 20052005.

[6] K. Hammani, et al., "Optical rogue-wave fluctuations in fiber Raman amplifiers," Opt. Express, vol. 16, pp. 16467-16474, 2008.

[7] J.-F. Zhang, et al., "Self-similar parabolic pulses in optical fiber amplifiers with gain dispersion and gain saturation," Phys. Rev. A, p. 055801, 2008.

[8] B. G. Bale and S. Wabnitz, "Strong spectral filtering for a mode-locked similariton fiber laser," Opt. Lett., vol. 35, pp. 2466-2468, 2010.

[9] A. Heidt, et al., "Coherent octave spanning near-infrared and visible supercontinuum generation in allnormal dispersion photonic crystal fibers," Opt. Express, vol. 19, pp. 3775-3787, 2011.

[10] L. E. Hooper, et al., "Coherent supercontinuum generation in photonic crystal fiber with all-normal group velocity dispersion," Opt. Express, vol. 19, pp. 4902-4907, 2011.

[11] M. L. V. Tse, et al., "Supercontinuum generation at 1.06 um in holey fibers with dispersion flattened profiles," Opt. Express, vol. 14, pp. 4445-4451, 4/2006 2006.

[12] B. G. Bale and S. Boscolo, "Impact of third order dispersion on the evolution of parabolic optical pulses," J. Opt .A., vol. 12, p. 015202, 2010.

[13] C. Finot, et al., "Beneficial impact of wave-breaking or coherent continuum formation in normally dispersive nonlinear fibers," J. Opt. Soc. Am. B, vol. 25, pp. 1938-1948, 2008.

[14] S. Boscolo, et al., "Passive nonlinear pulse shaping in normally dispersive fiber systems," IEEE $J$. Quantum Electron., vol. 44, pp. 1196-1203, 2008.

[15] E. R. Andresen, et al., "Transform-limited spectral compression by self-phase modulation of amplitude shaped pulses with negative chirp," Opt. Lett., vol. 36, pp. 707-709, 2011. 